\title{
Evaluating a bound for MANETs routing protocols performance using graphs with activation windows
}

\author{
David Soler, Jose Albiach and Eulalia Martinez \\ Polytechnic University of Valencia \\ Dept. Applied Mathematics \\ Camino de Vera, s/n, 46071 Valencia, SPAIN \\ Email: dsoler@mat.upv.es
}

\author{
Pietro Manzoni \\ Polytechnic University of Valencia \\ Dept. Computer Engineering \\ Camino de Vera, s/n, 46071 Valencia, SPAIN \\ Email: pmanzoni@disca.upv.es
}

\begin{abstract}
In this paper we present an algorithm called STPA (Shortest Time Path Algorithm) which aims at providing a comparison tool for the evaluation of a bound for Mobile Ad Hoc Networks (MANETs) routing protocols performance.

STPA provides an exhaustive evaluation of an ideal routing protocol. Based on the current position and state of the nodes it can determine factors like: how many complete messages get to the destination, which is the smallest amount of time required by a packet to get to the destination, which path followed each packet, and so on. This values would allow a protocol designer to improve or fine tune his proposal.

We demonstrate that the complexity of the algorithm is $O\left(\sigma^{2}\right)$, that is polynomial with respect to parameter $\sigma$; where $\sigma$ corresponds to the sum of all the instants of time during which all nodes are active.
\end{abstract}

\section{INTRODUCTION}

Mobile ad-hoc networks (MANETs) are networks where mobile nodes can roam around at will and communicate with each other without any preexisting communication infrastructure.

A routing path has to be established in a multi-hop manner, requiring each mobile host to serve as a router. This fact creates many challenging research issues since collaborating nodes might have different capacities, like processing power, memory availability or willingness to be part of a route. This last factor, the willingness to be part of a route might depend on aspects such as the need for a node to be left physically turned off to save energy [1], or to simply make this node invisible, i.e., logically turned off, to certain other nodes for security reasons [2]. The effect of the presence of nodes in the off-state must therefore be thoroughly evaluated.

The evaluation of a new proposal is usually performed with the aid of a network simulator, like for example the ns-2 [3]. Anyway, the need for a formal model to describe a MANET has been approached in many other different ways. In [4] the author use games theory to evaluate cooperation in ad hoc networks for energy optimization. In [5] a framework called MERIT is described that can be used to assess routing protocols in MANETs. It uses the novel concept of a shortest mobile path (SMP) in a mobile graph, a generalization of the shortest path problem for mobile environments. Finally, in [6] the authors propose a novel graph-based mobility model, which provides a more realistic movement than the random walk model by reflecting the spatial constraints in the real world.

In this work we propose an analytical model based on graphs with activation windows. Our proposal provides an exhaustive evaluation of an ideal routing protocol. This values will allow a protocol designer to improve or fine tune his proposal. An activation window indicates when a node is considered active or inactive. A node is active, i.e., it can either send or receive messages, during certain time windows inside $[0, T]$.

We describe an algorithm, called STP algorithm (STPA), that based on the current position and state of the nodes determines factors like: how many complete messages get to the destination, which is the smallest amount of time required by a packet to get to the destination, which path followed each packet, and so on.

We show that the complexity of the algorithm is $O\left(\sigma^{2}\right)$, that is polynomial with respect to parameter $\sigma$; where $\sigma$ corresponds to the sum of all the instants of time during which all nodes are active.

The rest of this paper is organized as follows. Section II describe the proposed network model. Section III defines the objectives and presents the details of the STP algorithm. Finally, some conclusion and the description of the future work are presented.

\section{MODEL DEFINITION}

We represent an ad hoc network as a set of $n$ nodes, $W=$ $\left\{v_{i}\right\}_{i=1}^{n}$, each referring to a device placed on the $\mathbb{R}^{2}$ plane. We define a coordinate function:

$$
c o_{i}:[0, T] \rightarrow \mathbb{R}^{2}, \quad c o_{i}(t)=\left(x_{i}(t), y_{i}(t)\right)
$$

where $[0, T]$ is the total interval of time during which there is network activity. The coordinate function provides the position of node $v_{i}$ at any instant of time $t \in[0, T]$.

Each node $v_{i}$ can be either active or inactive. A node is active, i.e., it can either send or receive messages, during certain time windows inside $[0, T]$, where we will suppose that time takes integer values (discretized time). The set of time windows is defined as:

$$
t w_{i}=\left\{\left[t_{2 k-1}^{i}, t_{2 k}^{i}\right]\right\}_{k=1}^{p_{i}}
$$


where $0 \leq t_{s}^{i}<t_{r}^{i} \leq T$ if $s<r$ with $s, r \in\left\{1,2, \ldots, 2 p_{i}\right\}$. Node $v_{i}$ can only send messages at time $t$, where $t \in$ $\left[t_{2 k-1}^{i}, t_{2 k}^{i}\right]$ for some $k \in\left\{1, \ldots, p_{i}\right\}$. Outside $t w_{i}$ node $v_{i}$ is inactive and it can neither send nor receive any message.

The transmission range of a node is a function of the node itself and the sending time $t$. We use a generic range function, $R_{i}(t)$, defined as:

$$
R_{i}:[0, T] \rightarrow \mathbb{R}^{+} \cup\{0\}
$$

so that $R_{i}(t)=0, \forall t \notin \bigcup_{k=1}^{p_{i}}\left[t_{2 k-1}^{i}, t_{2 k}^{i}\right]$. This function can depend on many factors, like the RF technology adopted, the surrounding environment, the antenna type, and so on. If node $v_{i}$ sends a message at time $t$, and node $v_{j}$ is inside $v_{i}$ 's range at that instant of time, that is, $\left\|c o_{i}(t)-c o_{j}(t)\right\| \leq R_{i}(t)$, the message will reach $v_{j}$ at instant $t+t_{i j}(t)$.

In this paper we will us the term "packet" to indicate a message that requires a unit of time to leave a node. A packet is therefore the message unit and we will suppose that all messages consist of one or several packets and that at any instant of time a node can send at the most one packet.

Function $t_{i j}(t) \in \mathbb{Z}^{+} \cup\{0\}$ indicates the units of time it takes for a message to move from $v_{i}$ to $v_{j}$. The node $v_{j}$ will receive this message only if active, that is only if $t+t_{i j}(t) \in\left[t_{2 k-1}^{j}, t_{2 k}^{j}\right]$ for some $k \in\left\{1, \ldots, p_{j}\right\}$. We consider that $t_{i j}(t)$ is small enough so that $c o_{j}(t) \approx \mathrm{co}_{j}\left(t+t_{i j}(t)\right)$, i.e., that during that period of time $v_{j}$ stays inside the range of $v_{i}$, if $\left\|c o_{i}(t)-c o_{j}(t)\right\| \leq R_{i}(t)$. We are basically supposing that, since sending time are so small compared to the nodes mobility, vertices are practically static during each sending operation.

The sending of a message from $v_{i}$ at time $t$ and the reception at node $v_{j}$ at instant $t+t_{i j}(t)$ if $v_{j}$ is in the $v_{i}$ range, implies a cost $c_{i j}(t) \geq 0$. We consider in this work that the cost coincides with the sending time $t_{i j}(t)$.

Once node $v_{j}$ receives the message from $v_{i}$ and determines that it has to forward it, it will do it at time $t+t_{i j}(t)+r_{j}$, where function $r_{j} \in \mathbb{Z}^{+} \cup\{0\}$ represents the processing time at node $v_{j}$. The instant of time $t+t_{i j}(t)+r_{j}$ must belong to the same activation window of $t+t_{i j}(t)$ and of $v_{j}$, otherwise the message will get lost at $v_{j}$.

We will suppose that in the network the following circumstances are verified:

- If a node $v_{i}$ receives more than one packet at the same instant of time, because of interferences all the packets will be lost in $v_{i}$.

- A node does not forward a packet originated by itself nor resends a packet whose destination is itself, nor resends a packet sent previously by itself. Basically a packet is resent by a node only if it comes from different paths, thus avoiding loops.

- To save energy, a node $v_{i}$ resends a packet at most $g_{i}$ times, ignoring all packets coming after the $g_{i}$-th resending operation.

- If a node $v_{j}$ is about to send a locally generate packet at instant $t$, but at the instant $t-r_{j}$ receives one and only one packet, except in the three cases listed below, it will send the packet received at instant $t$ and it will send its own packet in the following available instant $t$, delaying if it is required any other own packets. If at instant $t-r_{j} v_{j}$ receives more than one packet, as explained before these packets will be lost and therefore the node will send its own packet at instant $t$. The three exceptions are:

- If the packet that receives at $t-r_{j}$ is directed to it;

- if a packet was sent by $v_{j}$ in a previous instant of time;

- if the packet received at instant $t-r_{j}$ is not directed to $v_{j}$ and it already forwarded it $g_{i}$ times;

in the above three cases it will send its own packet at instant $t$.

\section{The STPA Algorithm}

This section describes the details of the proposed algorithm. We first formally describe the problem, then we give the details of the algorithm, and finally we compute its complexity.

\section{A. Problem statement}

We suppose an ad hoc network made of $n$ nodes, $W=$ $\left\{v_{i}\right\}_{i=1}^{n}$. About this network we know all the information mentioned in Section II referring to an interval of time $[0, T]$. We suppose that during interval $[0, T]$ the subset of nodes $\left\{v_{i_{j}}\right\}_{j=1}^{r}$, where $r<n$ and $i_{j}<i_{j+1}$ $\forall j$, generate (i.e., send) messages. That is, node $v_{i_{j}}$ send $m_{i_{j}}$ messages $M_{1}^{i_{j}}, M_{2}^{i_{j}}, \ldots, M_{m_{i_{j}}}^{i_{j}}$ respectively to nodes $v_{1_{i_{j}}}, v_{2_{i_{j}}}, \ldots, v_{m_{i_{j_{j}}}}$ which must not be two disjunct. Each message $M_{k}^{i_{j}}$ has a unique destination and it is made of $p_{k}^{i_{j}}$ packets sent at instants of time $t_{1}^{k_{i_{j}}}, t_{2}^{k_{i_{j}}}, \ldots, t_{p_{k}^{i_{j}}}^{k_{i_{j}}}$ where $0 \leq t_{1}^{k_{i_{j}}}<t_{2}^{k_{i_{j}}}<\ldots<t_{p_{k}^{i_{j}}}^{k_{i_{j}}}<T$. Packets related to different messages can be alternated, like for example, $t_{1}^{k_{i_{j}}}<t_{1}^{h_{i_{j}}}<t_{2}^{k_{i_{j}}}$ supposing $h \neq k$, where node $v_{i_{j}}$ after sending the first packet of message $M_{k}^{i_{j}}$ send the first packet of message $M_{h}^{i_{j}}$ and afterward the second packet of message $M_{k}^{i_{j}}$.

The answers that we will give with our approach are all oriented to offer criteria to determine how effective is the routing protocol we are proposing with respect to an "ideal" protocol. More specifically we will obtain:

- how many complete messages get to the destination;

- how many complete in-order messages get to the destination;

- which packets will be delayed and for how long;

- at which instant of time will each successful packet get to its destination;

- which is the smallest amount of time required by a packet to get to the destination;

- which path was followed by each packet.

\section{B. The algorithms details}

By default all variables that are not initially set to a different value must be considered as initialized at 0 . 
Each vertex $v_{m}^{h}$ that appears in the graph refers to node $v_{m}$ at the instant of time $h$. Associated to each vertex $v_{m}^{h}$ there is a 4-component vector $t e_{m}^{h}=\left(\tilde{m}, \tilde{h}, \hat{m}, \tilde{h}^{\prime}\right)$. This vector must be interpreted as if node $v_{m}$ at instant $h$ forwards the packet sent for the first time by node $v_{\tilde{m}}$ at instant $\tilde{h}$, and whose destination is node $\hat{m}$; this packet was initially scheduled to be sent by $v_{\tilde{m}}$ at instant $\tilde{h}^{\prime}$. Note that $\tilde{h}>\tilde{h}^{\prime}$ means that the corresponding packet was sent with a delay of $\tilde{h}-\tilde{h}^{\prime}$ units of time.

The vertexes $v_{m}^{h}$ that generate packets, will sometime have a second associated vector, st $e_{m}^{h}$, similar to the previous one, and a third one, $t t e_{m}^{h}$. These two vectors are used to determine which packet will be eventually sent.

Each vertex $v_{m}^{h}$ will have another associated vector $\mathrm{cam}_{m}^{h}$, with at most $n-1$ components (where $n$ is the total number of nodes). The i-th component of $\operatorname{cam}_{m}^{h}$ will indicate the i-th node through which passed the packet that $v_{m}$ will possibly send at instant $h$. This vector is used to avoid loops.

Obviously, at the beginning $\operatorname{cam}_{m}^{h}=\emptyset$ for all $m$ and $h$. The first element of $\mathrm{cam}_{m}^{h}$, when created, indicates the node that generated the packet.

In the algorithm description we will use the symbol $\oplus$ to indicate the addition of a component to vector $\mathrm{cam}_{m}^{h}$; e.g., $(2,3) \oplus 4=(2,3,4)$. Also, $c \in \operatorname{cam}_{m}^{h}$ will indicate that one of the components of $c a m_{m}^{h}$ is node $c$; therefore $m \in c_{a m}^{h}$ will alert about the creation of a loop.

Each vertex $v_{m}^{h}$ will have associated a binary variable $e t i q_{m}^{h}$ which will be set to 1 if node $v_{m}$ receives 2 or more packets at instant $h-r_{m}$. This variable is associated only to vertexes that do not generate packets. Vertexes $v_{m}^{h}$ that generate packets will have associated another variable $r e c_{m}^{h}$ which will be set to 0 if node $v_{m}$ does not receive any packet at instant $h-r_{m}$, or to 1 if it receives a unique packet at instant $h-r_{m}$, or to 2 if it receives two or more packets at instant $h-r_{m}$ (interferences). All the etiq and rec variables will be set to 0 at the beginning.

Using $c\left(v_{m}^{h}, v_{m^{\prime}}^{h^{\prime}}\right)$ we will indicate the cost of arc $\left(v_{m}^{h}, v_{m^{\prime}}^{h^{\prime}}\right)$ in $G$; in this work this will always coincide with $h^{\prime}-h$, that is with $t_{m m^{\prime}}(h)+r_{m^{\prime}}$. By $d^{+}(v)$ we will understand, as normal in graph theory, the number of arcs that comes out from vertex $v$. Finally a set $N$ will contain all those $t e_{m}^{h}$ vectors that correspond to packets that could not be sent since their sending instant were delayed to an instant at which the node is inactive and will remain inactive until $T$.

Basically the algorithm starts from an initial set $Q$ with all the vertexes $v_{m}^{h}$ so that $v_{m}$ belongs to the subset of nodes that generate their own messages and $h$ is the instant of time at which $v_{m}$ wants to send one of its packets; therefore $Q$ will initially have as much vertexes as many locally generated packets that the nodes want to send in the interval $[0, T]$.

At each iteration the algorithm searches in strict increasing time order a vertex $v_{a}^{b}$ in $Q$, and it eliminates it from $Q$, looking for all nodes $v_{m}$ and instants of time $b+t_{a m}(b)$ where, given the network conditions, it is guaranteed that the message sent from $v_{a}$ at instant $b$ will get to $v_{m}$.

For the cases where this guarantee exists, the algorithm adds the vertexes $v_{m}^{b+t_{a m}(b)+r_{m}}$ to $V$ and $Q$ with their respective

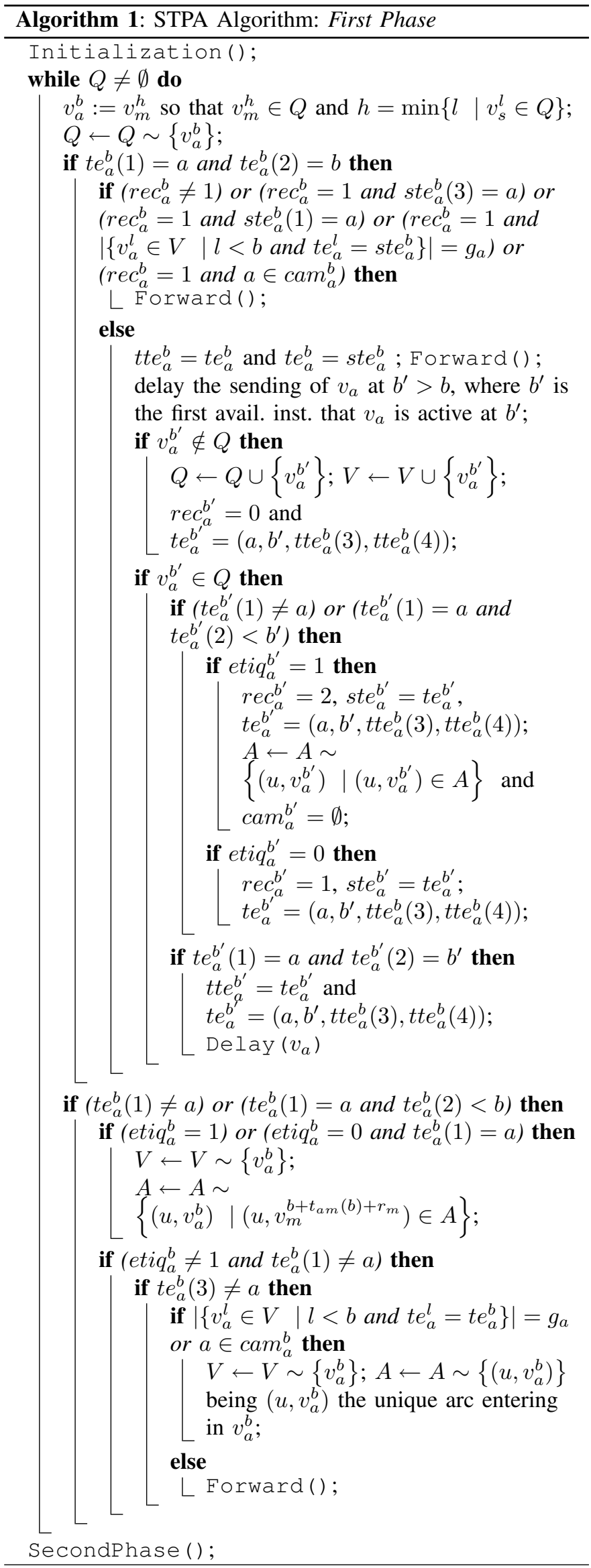




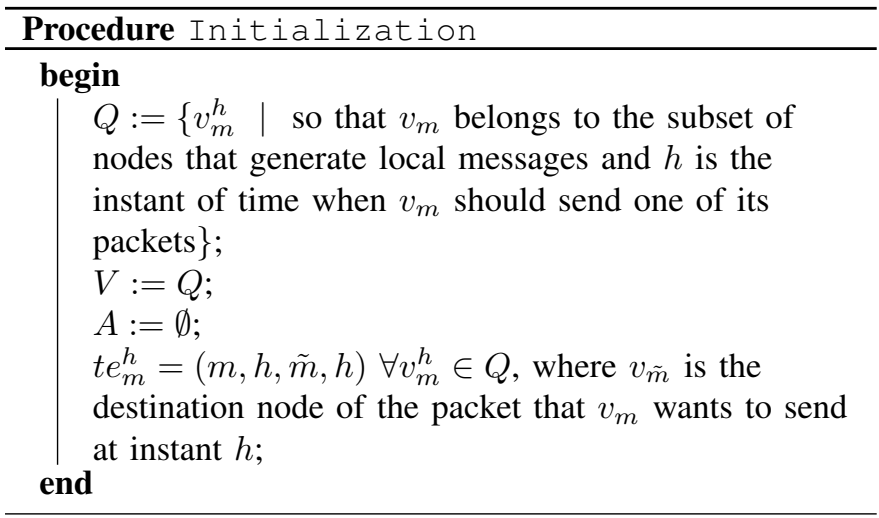

vectors $t e_{m}^{b+t_{a m}(b)+r_{m}}$, and the arcs $\left(v_{a}^{b}, v_{m}^{b+t_{a m}(b)+r_{m}}\right)$ to $A$, or it properly mark them if they already belong to $Q$, depending on node $v_{m}$ receives at instant $b+t_{a m}(b)$ two or more packets and therefore, due to interferences, it loses the information of those packets or it generate a local packet at instant $b+t_{a m}(b)+r_{m}$.

If the third vector component of the selected vertex $v_{a}^{b}$ is $a$ (i.e., $t e_{a}^{b}(3)=a$ ), means that $v_{a}$ is the destination of the received packet, then $v_{a}^{b}$ will be eliminated from $Q$ without any further search, unless $v_{a}$ generates at the same instant of time a local packet at $t=b$. Iteration stops when $Q=\emptyset$.

Finally, to reduce as much as possible the size of $G$ we eliminate from $V$ in strict decreasing time order all those vertexes $v_{m}^{h}$ without leaving $\operatorname{arcs}\left(d^{+}\left(v_{m}^{h}\right)=0\right)$ where $v_{m}$ does not generate any local packet at instant $t=h$ and $t e_{m}^{h}(3) \neq m$, which means that $v_{m}$ is not the destination of the received packet at instant $h-r_{m}$, eliminating at the same time its input arc of $A$, which can generate more vertexes without any leaving arc in $G$ at previous instant. The resulting directed graph $G=(V, A)$ will be acyclic and will be formed by:

- Maximum paths, they are not part of a longer path, which go from vertexes $v_{m}^{h}$ so that $v_{m}$ belongs to the subset of nodes the generate their own messages and $h$ is the instant of time at which $v_{m}$ send one of its packets, to vertexes $v_{m^{\prime}}^{h^{\prime}}$ so that $v_{m^{\prime}}$ is the destination node of the packet originally send by $v_{m}$ at $t=h$.

- Various of the previous paths concatenated among them, if it happens that $v_{m^{\prime}}^{h^{\prime}}$ is at the same time generator of a local packet.

- Isolated vertexes that correspond to packets sent which did not get to their final destination.

Two paths corresponding to two different packets will be vertex-disjoint, except in the case that they are concatenated. In this last case they will have in common the last vertex of the former and the first vertex of the latter.

Thanks to how the algorithm is designed and to some basic properties of graph theory it is easy to demonstrate the following statements, which we have included in the following unique theorem:

Theorem 1: Given an ad hoc network, described with all the parameters of Section II and given the graph $G$ and the

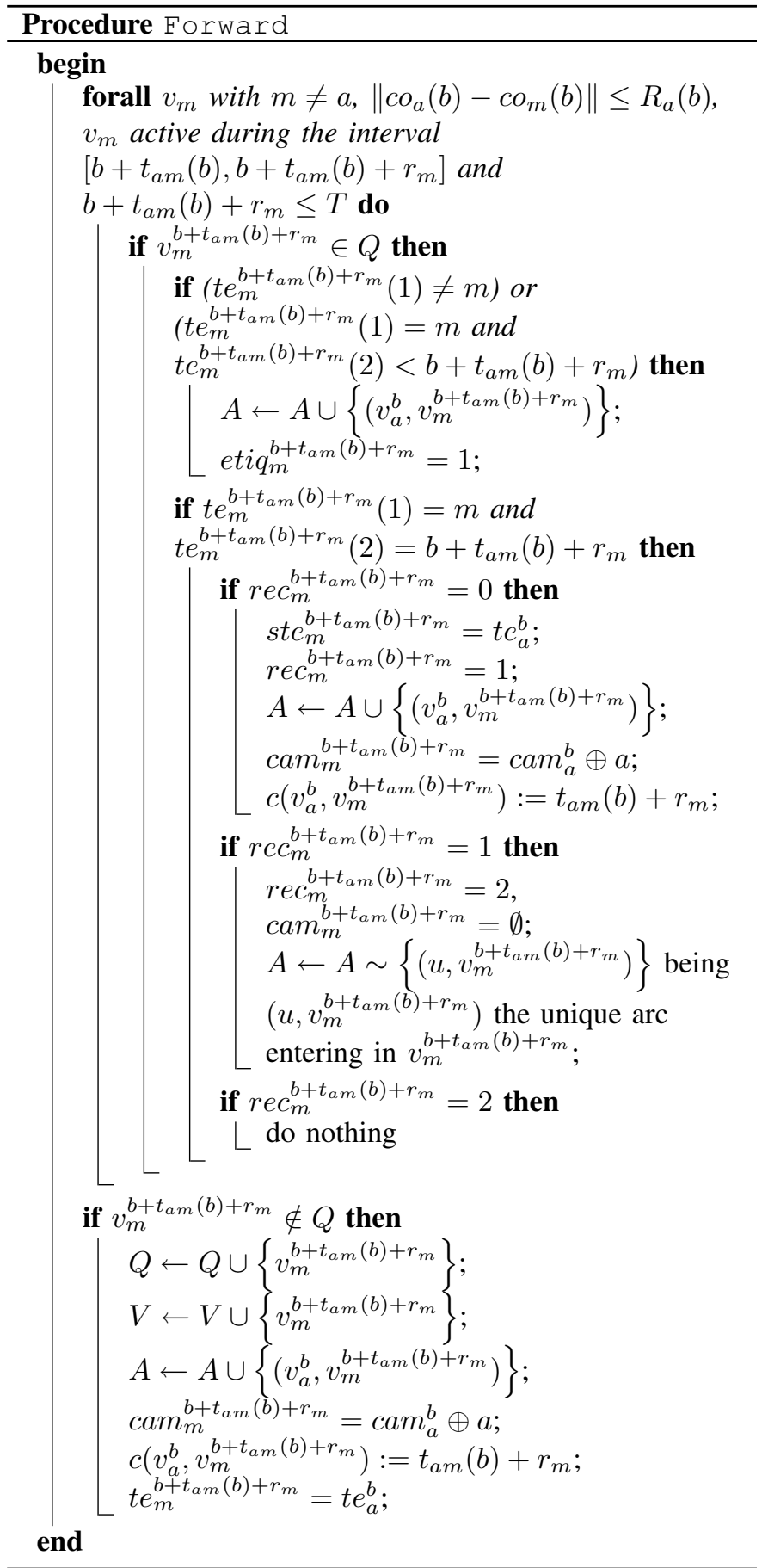

set of vectors $N$ obtained applying the STPA algorithm, it is verified that:

a) A packet generated and sent by $v_{s}$ at instant $t$ will not get to its destination if and only if $v_{s}^{t} \in V$ and $d^{+}\left(v_{s}^{t}\right)=0$.

b) A packet that was scheduled to be generated and sent by $v_{s}$ at instant $t$ to $v_{d}$, will be definitively sent by $v_{s}$ at instant $t^{\prime}$, where $t^{\prime} \geq t$ if $v_{s}^{t^{\prime}} \in V$ and $t e_{s}^{t^{\prime}}=$ $\left(v_{s}, t^{\prime}, v_{d}, t\right)$.

c) A packet that was scheduled to be generated and sent 

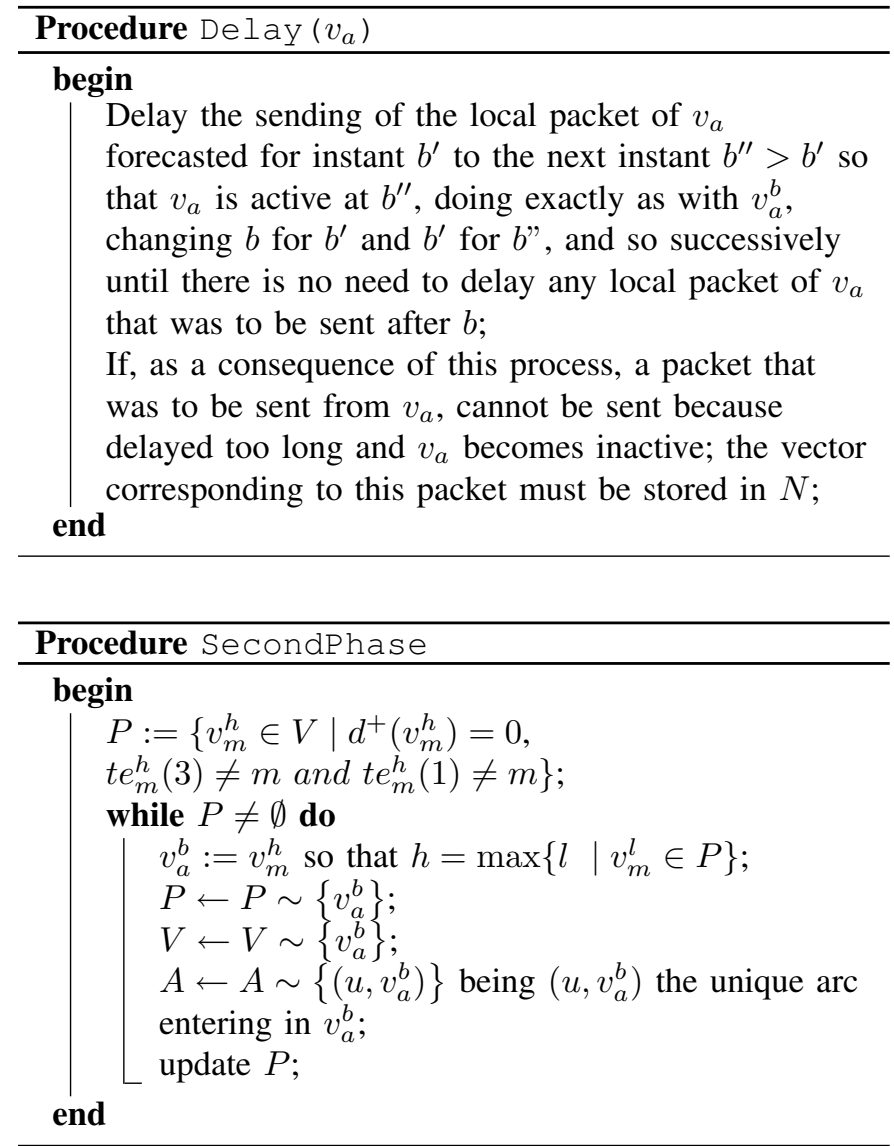

by $v_{s}$ at instant $t$ to $v_{d}$, will not be sent by $v_{s}$ at any instant of time if a vector $\left(v_{s}, t^{\prime}, v_{d}, t\right) \in N$ for some $t^{\prime} \geq t$.

d) If $v_{s}$ generates and sends a packet at instant $t$ to $v_{d}$, this packet will get to its destination at instant $t^{\prime}$ if it exists in $G$ a path from $v_{s}^{t}$ to $v_{d}^{t^{\prime}+r_{d}}$.

e) Given a maximum path in $G$ that starts from $v_{s}^{t}$ and ends in $v_{d}^{t^{\prime}}$, then either $t e_{s}^{t}(3)=v_{d}$ or two vertexes exist inside that path in $G, v_{i}^{h}$ and $v_{j}^{h^{\prime}}$ with $t<h \leq h^{\prime}<t^{\prime}$ (possibly $i=j$ and $h=h^{\prime}$ ) so that $t e_{s}^{t}(3)=v_{i}$ and $t e_{j}^{h^{\prime}}(3)=v_{d}$.

Thanks to the information stored into vectors $t e_{d}^{t}$ we know which, if any, packet gets at each instant to $v_{d}$. For example, if we want to know at which instant the packet sent from $v_{s}$ at instant $m$ will get to $v_{d}$, we will have to look for the vertex $v_{d}^{h}$ in $V$, if it exists, so that $h=\min \left\{l \mid v_{d}^{l} \in V\right.$ and $\left.t e_{d}^{l}=\left(s, m, d, m^{\prime}\right)\right\}$. This packet will get to $v_{d}$ at instant $h-r_{d}$. The time required by the packet since it left $v_{s}$ at instant $m$ will be $h-r_{d}-m$ time units. It was initially scheduled to be sent at $m^{\prime}$, where $m>m^{\prime}$, meaning that the packet suffered a delay of $m-m^{\prime}$ time units. The information about the path followed by the packet is stored in $c a m_{d}^{h}$. By applying a simple method of graph theory to compute the unique path from $v_{s}^{m}$ to $v_{d}^{h}$ in $G$ and checking the super-indexes of the vertexes in the path we can determine at which instant of time the packet has been forwarded by each node in the path.
Item $e$ of the previous theorem means that given a maximum path in $G$ that starts from $v_{s}^{t}$ and ends in $v_{d}^{t^{\prime}}$, two situations can occur: either $v_{s}$ generates and send a packet at instant $t$ to $v_{d}$, which will receive it at $t^{\prime}-r_{d}$, or this maximum path is made of various concatenated paths, as if for example node $v_{i}$ receives a packet being the final destination at instant $h-r_{i}$ and at the same time it generates and send a packet at instant $h$ (vertex $v_{i}^{h}$ inside the path).

\section{Complexity computation of STPA}

In this section we determine the upper bound for the complexity of the STPA algorithm. We will not minimize this upper bound but we will show that the complexity of the algorithm is polynomial with respect to parameter $\sigma$. This parameter corresponds to the sum of all the instants of time during which all nodes are active, that is:

$$
\sigma=\sum_{i=1}^{n} \sum_{j=1}^{p_{i}}\left(t_{2 j}^{i}-t_{2 j-1}^{i}\right)
$$

If we define $\sigma_{i}=\sum_{j=1}^{p_{i}}\left(t_{2 j}^{i}-t_{2 j-1}^{i}\right)$ where $i \in$ $\{1,2, \ldots, n\}$, we can redefine the previous definition of $\sigma$ as: $\sigma=n \bar{\sigma}$ where $\bar{\sigma}$ is the average value of all $\sigma_{i}$, that is of the instants of time at which each node is active in $[0, T]$.

Theorem 2: The STPA algorithm has complexity $O\left(\sigma^{2}\right)$.

Proof:

The number of iterations made by the algorithm in the first phase is bounded above by $\sigma$ since at each iteration it sets a different $v_{s}^{t}$ and it eliminates it from $Q$. At each iteration, the number of comparisons required to look for the selected vertex is also bounded above by $\sigma$, since $|Q| \leq \sigma$.

Once $v_{a}^{b}$ is selected, the procedures that impact the complexity of the algorithm are:

- Compute $\mid\left\{v_{a}^{l} \in V \quad \mid l<b\right.$ and $\left.t e_{a}^{l}=s t e_{a}^{b}\right\} \mid$, with a number of basic operation $O\left(\sigma_{a}\right)$ (we have to check a number of instants $l$ bounded by $\sigma_{a}$ ).

- The process of, if it is the case, delaying the sending of a locally generated packet, requires also $O\left(\sigma_{a}\right)$ basic operations. These operations will be done at an instant of time after $b$ when $v_{a}$ is active.

- The subroutine FORWARD requires just a few operations for each node $v_{m}$ where $m \neq a$, basically checking if it is active over the interval $\left[b+t_{a m}(b), b+t_{a m}(b)+r_{m}\right]$. The value $r_{m}$ should be very small, we consider the bound for this interval width equal to $\sigma_{m}$, and therefore the complexity of this subroutine is $O(\sigma)$.

Regarding the second phase, a few checks must be done for each vertex $V$, where $|V| \leq \sigma$ to determine $P$. $P$ will have less vertexes than $|V|$, vertexes which will require just a few basic operations. Therefore the complexity of this second phase is $O(\sigma)$ and the total complexity of the algorithm is $O\left(\sigma^{2}\right)$. 


\section{CONCLUSiOnS}

In this paper we presented an algorithm called STPA (Shortest Time Path Algorithm) which aims at providing a comparison tool for the design of routing algorithm for Mobile Ad Hoc Networks (MANETs).

Instead of validating new proposals by using simulation with typical tools like ns2, what STPA provides is an exhaustive evaluation of an ideal routing protocol. Based on the current position and state of the nodes it can determine factors like: how many complete messages get to the destination, which is the smallest amount of time required by a packet to get to the destination, which path followed each packet, and so on. This values would allow a protocol designer to improve or fine tune his proposal.

We demonstrate that the complexity of the algorithm is $O\left(\sigma^{2}\right)$, i.e., polynomial with respect to parameter $\sigma$; where $\sigma$ corresponds to the sum of all the instants of time during which all nodes are active.

This is anyway a preliminary work. We are currently designing a software tool that will exhaustively evaluate a given scenario and that will provide all the characterizing parameters of an ideal routing protocol.

Mobility will also be introduced to completely evaluate MANETs scenarios.

\section{ACKNOWLEDGMENTS}

This work was partially supported by FEDER and the Ministerio de Educación y Ciencia, Spain, under Grant TIN200507705-C02-01.

\section{REFERENCES}

[1] Juan Carlos Cano and Pietro Manzoni. Evaluating the energyconsumption reduction in a MANET by dynamically switching-off network interfaces. Proceedings of the 6th IEEE Symposium on Computers and Communications, Hammamet, Tunisia, July 2001.

[2] Hao Yang, James Shu, Xiaoqiao Meng, and Songwu Lu. Scan: Selforganized network-layer security in mobile ad hoc networks. IEEE Journal on Selected Areas in Communications, 24(2):261-273, 2006.

[3] K. Fall and K. Varadhan. ns notes and documents. The VINT Project. UC Berkeley, LBL, USC/ISI, and Xerox PARC, February 2000. Available at http://www.isi.edu/nsnam/ns/ns-documentation.html.

[4] Sonja Buchegger and Jean-Yves Le Boudec. Performance analysis of the CONFIDANT protocol: Cooperation of nodes fairness in distributed ad-hoc networks. In Proceedings of IEEE/ACM MobiHOC, 2002.

[5] A. Faragó and V. R. Syrotiuk. Merit: a scalable approach for protocol assessment. Mob. Netw. Appl., 8(5):567-577, 2003.

[6] Jing Tian, Joerg Haehner, Christian Becker, Illya Stepanov, and Kurt Rothermel. Graph-based mobility model for mobile ad hoc network simulation. In 35th Annual Simulation Symposium, April 14 - 18, 2002, San Diego, California. 